\title{
Oral Amoxicillin-Clavulanic Acid Treatment in Urinary Tract Infections Caused by Extended-Spectrum Beta-Lactamase-Producing Organisms
}

\author{
Ali Beytur ${ }^{1,}$; Yusuf Yakupogullari ${ }^{2}$; Fatih Oguz ${ }^{1}$; Baris Otlu ${ }^{2}$; Halim Kaysadu ${ }^{2}$ \\ ${ }^{1}$ Urology Clinic, Inonu University Medical Faculty, Malatya, Turkey \\ ${ }^{2}$ Department of Medical Microbiology Inonu University Medical Faculty, Malatya, Turkey \\ *Corresponding author: Ali Beytur, Urology Clinic, Inonu University Medical Faculty, Malatya, Turkey. Tel: +90-5326207996, E-mail: ali.beytur@inonu.edu.tr
}

Received: July 26, 2013; Revised: November 11, 2013; Accepted: December 16, 2013

\begin{abstract}
Background: Extended-spectrum $\beta$-lactamases (ESBLs) are increasing problems. The involvement of ESBL-producing organisms is associated with higher rates of carbapenem usage in urinary tract infections (UTIs). Though some strains are susceptible to amoxicillin-clavulanic acid (AMC) in vitro, there is very less data about the consequences of AMC usage for such infections.

Objectives: The purpose of this study was to evaluate the clinical and microbiological outcomes of AMC treatment in UTIs caused by AMC-susceptible ESBL-producing organisms.

Patients and Methods: A retrospective cohort study was conducted in a tertiary care hospital. Forty-six out of 652 patients (F/M ratio: 32/14; mean age: 43.9 years) with ESBL-positive UTIs were eligible for this study. These patients had cystitis $(n=23)$, vesicoureteral reflux $(n=7)$, hyperactive bladder $(n=6)$, and prostatitis $(n=10)$. Data was collected via chart review and was statistically analyzed.

Results: AMC-susceptible ESBL-producing Escherichia coli, Klebsiella pneumoniae and K. oxytoca were identified as the causative agents in 31,14, and 1 patients, respectively. Thirty-nine (84.7\%) out of 46 patients were successfully treated with oral AMC. Additionally, 2 (4.3\%) patients' urine cultures turned to be negative, though their clinical complaints and leukocyturia had continued. In the remaining $5(10.8 \%)$ patients, no positive clinical and microbiological response was obtained. Increased minimum inhibitory concentration levels of AMC (from 4 to $>256 \mu \mathrm{g} / \mathrm{mL}$ ) were detected in these patients and the treatment failures were attributed to this developing resistance. We found that therapeutic failure was significantly more frequent in Klebsiella spp. than in E. coli (33.3\% vs $6.5 \%, \mathrm{P}=0.029)$. Furthermore, no treatment failure was observed in pathogens with minimum inhibitory concentrations (MICs) $\leq 2 \mathrm{mg} / \mathrm{mL}$, and the high AMC MIC $(8 \mathrm{mg} / \mathrm{mL})$ was associated with resistance development and therapeutic failure (71.4\% vs. 5.1\%, $\mathrm{P}=0.0001)$.

Conclusions: Our results suggested that amoxicillin-clavulanic acid may be a good oral antimicrobial which can be used for treatment of ESBL-positive UTIs, if the causative agent is susceptible to this antibiotic. However, some strains may develop resistance during therapy, especially in those exhibiting high AMC MICs.
\end{abstract}

Keywords:Urinary Tract Infection; beta-Lactamase; Amoxicillin-Clavulanic Acid

\section{Background}

Urinary tract infections (UTIs) are among the most frequent infections acquired in the community and in hospitals. It is estimated that $>20 \%$ of females get UTI in their lifetime and this rate may increase to $30-40 \%$ in elderly males and females due to prostatic and gynaecological surgeries (1). Three decades after their emergence, extended-spectrum $\beta$-lactamases (ESBLs) have become one of the most important drug resistance mechanisms for common uropathogens, including Escherichia coli and Klebsiella pneumoniae. In 2011, the European Centre for Disease Control and Prevention stated that the prevalence of ESBL-positive E. coli has significantly increased in more than half of the $28 \mathrm{Eu}$ ropean countries over the past four years (2). Formerly, the Clinical and Laboratory Standards Institute (CLSI)
(3) had recommended avoidance from many classes of $\beta$-lactams, including penicillins, cephalosporins and monobactams for treatment of ESBL-positive infections, regardless of the susceptibility test results. Though this suggestion has recently changed (4), conflict still exists, particularly on $\beta$-lactam plus $\beta$-lactamase inhibitor combinations. Furthermore, there is less data regarding the clinical success of these drugs.

\section{Objectives}

The purpose of this study was to assess the clinical and microbiological outcomes of ESBL-positive UTIs in patients treated with amoxicillin-clavulanic acid (AMC), in which the infecting organism was susceptible to this drug in vitro. 


\section{Patients and Methods}

\subsection{Study Design}

A retrospective cohort study was conducted with the collaboration of the urology clinics of a university hospital and a state hospital in Malatya City, Turkey, between September 2010 and October 2013. A total of 652 patients with UTIs caused by ESBL-producing organisms were reviewed. Of these, 81 patients who were infected with AMC-susceptible strains were identified, of which 46 were eligible for this study. Patients' data including age, gender and clinical progress were collected via chart review from the hospital data management system. This study was approved by the Inonu University Human Ethical Committee (ref No.: 2010/76).

\subsection{Definitions}

The cases with clinical symptoms (dysuria, urgency and frequency), pyuria, and significant bacterial growth ( $\geq 105$ $\mathrm{cfu} / \mathrm{mL}$ ) on culture were diagnosed as UTIs. Positive clinical response was defined as complete resolution of the symptoms and signs of infection. Negative clinical response was defined as no improvement or deterioration of these signs and symptoms despite treatment. Positive microbiological response was considered if the organism could not be isolated in repeat cultures during or after the course of AMC therapy. Negative microbiological response was defined as the continuation of pathogen growth on the bacterial culture despite appropriate antimicrobial treatments.

\subsection{Urinalyses and Culture}

Midstream urine samples were collected aseptically and urinalyses including biochemical measurements, microscopic examination and bacteriologic culture were performed for each patient's sample. The collected samples were inoculated on blood agar and eosin-methylene blue agar media (Oxoid, UK) using a standard loop. The bacterial strains were identified using standard microbiological methods. Antimicrobial susceptibility and ESBL production of the organisms were investigated using the disc diffusion method and the double-disc synergy test, respectively, according to CLSI guideline (4). The minimum inhibitory concentration (MIC) of AMC for the studied strains was measured using E-test strips (BioMerieux, France), according to the manufacturer's protocol. Fisher's exact test was used to analyze the data. $\mathrm{P}<0.05$ was accepted as statistically significant.

\section{Results}

In total, 46 patients who had UTIs due to AMC-susceptible ESBL-positive organisms between September 2010 and October 2013 were studied. Thirty-two of the patients were female and 14 were male. The mean age of the patients was 43.9 years (range: 4-85). Cystitis was diagnosed in 23 patients, vesicoureteral reflux in seven patients, six patients had hyperactive bladder, and 10 had prostatitis. E. coli $(\mathrm{n}=$ 31) was the most frequent causative organism of the UTIs, followed by K. pneumoniae $(\mathrm{n}=14)$ and K. oxytoca $(\mathrm{n}=1)$. After the AMC treatment, no bacterial growth (positive microbiological response) was detected in 41 of the patients (89.1\%), while significant bacteriuria continued in the other $5(10.8 \%)$. Thirty-nine patients $(84.7 \%)$ reported complete resolution of UTI symptoms after the treatment (clinical cure). Persistent clinical complaints were recorded in two patients despite of AMC management, thought their urine cultures yielded no growth (positive microbiological response but no clinical response). In five patients whose urine cultures remained positive after the treatment, no clinical improvement was also reported. Isolate distribution and therapy responses are shown in Table 1.

The MIC distribution of AMC for the tested strains was in the $0.5-8 \mu \mathrm{g} / \mathrm{mL}$ range. The MIC50 was 2 and the MIC90 was $8 \mu \mathrm{g} / \mathrm{mL}$. MIC values of the strains are shown in Table 2. Statistical analysis showed that higher MICs of AMC ( $8 \mu \mathrm{g} /$ $\mathrm{mL}$ ) were associated with therapeutic failure (71.4\% vs. $5.1 \%$, $\mathrm{P}=0.0001)$ and no therapeutic failure was observed in the pathogens with $\leq 2 \mu \mathrm{g} / \mathrm{mL}$ AMC MIC. Additionally, therapeutic failure was significantly more prevalent in Klebsiella spp. Than in E. coli (33.3\% vs. 6.5\%, $\mathrm{P}=0.029)$. Clinical responses according AMC MICs of the infecting organisms are shown in Figure 1.

\section{Discussion}

The conventional therapy of UTIs consists of antibiotic administration for 3-10 days. However, treatment of these infections is sometimes problematic due to the emergence of ESBL-mediated resistance among common uropathogens including E. coli and K. pneumoniae. Plasmids encoding ESBL genes frequently contain co-resistance determinants for aminoglycosides, sulphonamides and quinolones. Regarding their excellent activity against ESBL producers, carbapenems have been suggested as the most reliable drugs for the treatment of infections caused by such pathogens (5).

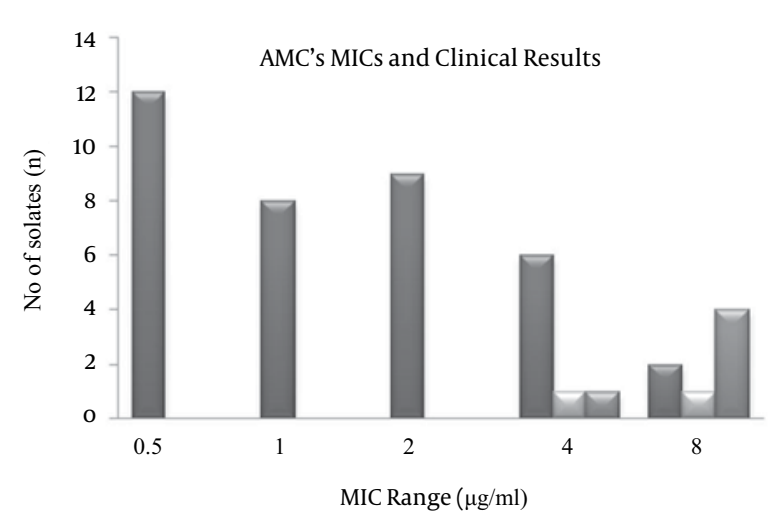

@ Positive CIinial Response $₫$ negative Microbiologic Response® Relaps

Figure 1. AMC MIC Values and Treatment Outcomes 
Beytur A et al.

\begin{tabular}{|c|c|c|c|}
\hline \multirow[t]{2}{*}{ Infection Type } & \multicolumn{3}{|c|}{ Therapy Responses } \\
\hline & Microbiologic response & Clinical cure & No response \\
\hline \multicolumn{4}{|l|}{ Cystitis ( $(n=23)$} \\
\hline E. coli & 15 & 15 & \\
\hline K. pneumoniae & 6 & 5 & 1 \\
\hline K. oxytoca & 1 & & \\
\hline \multicolumn{4}{|c|}{ Vesicoureteral reflux-cystitis $(n=7)$} \\
\hline E. coli & 4 & 4 & 1 \\
\hline K. pneumoniae & 1 & 1 & 1 \\
\hline \multicolumn{4}{|l|}{ K. oxytoca } \\
\hline \multicolumn{4}{|c|}{ Hyperactive bladder-cystitis $(n=6)$} \\
\hline E. coli & 4 & 4 & \\
\hline K. pneumoniae & 2 & 2 & \\
\hline \multicolumn{4}{|l|}{ K. oxytoca } \\
\hline \multicolumn{4}{|c|}{ Prostatitis $(n=10)$} \\
\hline E. coli & 7 & 7 & 1 \\
\hline K. pneumoniae & 1 & 1 & 1 \\
\hline \multicolumn{4}{|l|}{ K. oxytoca } \\
\hline Total & 41 & 39 & 5 \\
\hline
\end{tabular}

Table 2. Amoxicillin-Clavulanic Acid Minimum Inhibitory Concentrations of the Infecting Organisms ${ }^{a, b}$

\begin{tabular}{lcccc}
\hline MIC Values & \multicolumn{4}{c}{ AMC MICs $(\mu \mathrm{g} / \mathbf{m L})$} \\
\cline { 2 - 5 } & E. coli & K. pneumoniae & K. oxytoca & Overall \\
\hline MIC50 & 2 & 4 & 2 \\
MIC90 & 8 & 8 & 8 & $0.5-8$ \\
MIC Range & $0.5-8$ & $1-8$ & & \\
\hline a Abbreviations: & AMC, amoxicillin-clavulanic acid; MIC, minimum \\
inhibitory concentration. & & & \\
b Single isolate.
\end{tabular}

Therefore, their utilization has dramatically increased worldwide along with the spread of ESBL resistance, and consequently, excessive use of carbapenems due to the increasing incidence of ESBL-positive infections has resulted in the emergence of carbapenem resistance in Gram-negative enteric bacilli and other nosocomial pathogens (6-8).

The prevalence of ESBL-producing Enterobacteriaceae varies worldwide, but it is assumed to reach as high as $40 \%$ for E. coli and 90\% for K. pneumoniae in some countries (9). Today, ESBL-mediated resistance is not only limited to healthcare facilities; it has become a significant public health concern because of the spread of ESBL genes among the pathogens causing communityacquired UTIs (10). Increasing trends in ESBL prevalence and in the carbapenem resistance among Enterobacteriaceae have augmented the need for maximum expediency from the available alternative drugs such as AMC.
AMC is an aminopenicillin plus $\beta$-lactamase inhibitor combination which comes in both parenteral and oral forms. It can be prescribed for almost all age groups. It is a relatively safe drug during pregnancy (11) and is a relatively inexpensive antibiotic. Additionally, it has good tissue penetration and is mainly excreted from urine.

Weber et al. (11) reported that AMC reached 2-3 times higher urine concentrations than the effective MIC levels and remained at these concentrations in $>75 \%$ of the dosing intervals. Unlike many other penicillin plus sulbactam combinations and the first- and second-generation cephalosporins, this drug may be effective for some ESBL producers due to its clavulanate component. However, the clinical efficacy of AMC treatment remains unknown for many infection types that are caused by ESBL-producing organisms. In a recent study, RodriguezBano et al. (12) compared the impact of $\beta$-lactam plus $\beta$-lactamase inhibitor combinations with carbapenems on the outcome of bloodstream infections due to ESBL-producing E. coli in a post-hoc analysis. They did not find any significant difference in 30-day mortality between the patients treated with AMC or piperacillintazobactam (PTZ) and those treated with carbapenems. Therefore, the authors emphasized that AMC and PTZ were suitable alternatives for carbapenems for treating patients with serious infections due to ESBL-producing organisms if active in vitro, and would be particularly useful as a definitive therapy.

In some case reports, successful clinical outcomes have been reported regarding oral AMC treatment in prostatitis caused by ESBL-producing E. coli (13). Never- 
theless, the data regarding the outcomes of AMC treatment in the UTIs caused by ESBL producers is still limited. In this study, we evaluated the clinical results of oral AMC treatment in 46 patients with ESBL-positive UTIs. We observed that almost $90 \%$ of the patients' urine cultures became negative following the treatment and the majority of AMC-treated patients reported complete resolution of their clinical symptoms (Table 1). Urinalyses and culture confirmed that these patients fully recovered from their current UTIs. However, among the patients whose urine cultures turned to be negative, two patients reported persistence of the clinical symptoms. Significant leukocyturia was also present in these two patients. Therefore, their antimicrobial therapies were substituted with imipenem and both recovered. No positive clinical or microbiological responses were observed in the five patients, though they received adequate dose and time of AMC treatment.

In the follow-up cultures, MICs of AMC were found increased over the resistance breakpoints and we assumed that the therapeutic failures with these patients could be attributed to this developing resistance. When we considered the patients with therapeutic failure, we observed that four of five isolates of these patients had AMC MIC at $8 \mu \mathrm{g} / \mathrm{mL}$ and the remaining one strain had it at $4 \mu \mathrm{g} / \mathrm{mL}$. In the control culture that was performed after the treatment, we detected that AMC MICs of these five isolates increased to 128 or $\geq 256 \mu \mathrm{g} / \mathrm{mL}$. This possibly occurred because of the fact that AMC-resistant mutant strains might become predominant in the infection area, or overexpression of the ESBL enzyme could be stimulated, or the infecting agents might acquire additional resistance genes under AMC treatment. Similarly, the MICs of the strains isolated from the two patients who had positive microbiologic responses but no clinical response, were as high as $4 \mu \mathrm{g} / \mathrm{mL}$ and $8 \mu \mathrm{g} / \mathrm{mL}$. On the other hand, we determined that no resistance developed in the strains with AMC MIC $\leq 2 \mu \mathrm{g} / \mathrm{mL}$. Therefore, we thought that the risk of therapeutic failure was closely related to the increasing AMC MIC of the infecting organism (Figure 1).

In the patients with therapeutic failure, increased AMC MICs were detected in two E. coli and three K. pneumoniae strains after the treatment. Additionally, in two patients whose cultures were not clinically improved but the control cultures were negative, K. pneumoniae and $K$. oxytoca were the infecting agents (Table 1). Our results showed that positive clinical response could not be obtained in more than one third of the patients whose infecting agents were Klebsiella spp. Correspondingly, higher AMC MICs were detected in Klebsiella spp. isolates than in E. coli strains in our study (Table 2). Subsequently, we observed that therapeutic failure was more frequent in Klebsiella strains, most likely due to the fact that these agents had high MICs to AMC. In this study, we determined that AMC had a satisfactory clinical activity in the patients with UTIs caused by ESBL-producing
AMC-susceptible organisms. Therapeutic failure was observed in a few patients. Therefore, we believe that this drug may be a good therapeutic option for ESBL-positive UTIs treatment, if it is active in susceptibility testing. However, physicians must keep in mind that the infecting agent may develop resistance, especially if it has a high MIC.

\section{Authors' Contributions}

Ali Beytur: main organizer of this study; collected data, evaluated the patients and results, wrote the manuscript. Yusuf Yakupogullari co-organizer of this study; performed laboratory studies, partially wrote the manuscript. Fatih Oguz: co-organizer of this study; performed clinical investigations, partially wrote the manuscript. Baris Otlu: co-organizer of this study; performed laboratory studies, partially wrote the manuscript. Halim Kaysadu: co-organizer of this study; performed laboratory studies, partially wrote the manuscript.

\section{Funding/Support}

This study was financially supported by the Inonu University research fund (BAP: 2010-149).

\section{References}

1. Ryan KJ. Urinary Tract Infections. In: Ryan KJ, Ray CG editors. Sherris Medical Microbiology.. McGraw-Hill: New York; 2003. pp. 867-71.

2. European Center for Disease Prevention and Control.. Reporting on 2009 surveillance data and 2010 epidemic intelligence data Annual Epidemiological Report 2011. Stockholm; 2011.

3. Clinical and Laboratory Standards Institute.. Performance Standards for Antimicrobial Susceptibility Testing: Eighteen Informational Supplement, M100-S18. Wayne; 2008.

4. Clinical and Laboratory Standards Institute.. Performance Standards for Antimicrobial Susceptibility Testing: Eighteen Informational Supplement, M100-S18. Wayne; 2012.

5. Kizirgil A, Demirdag K, Ozden M, Bulut Y, Yakupogullari Y, Toraman ZA. In vitro activity of three different antimicrobial agents against ESBL producing Escherichia coli and Klebsiella pneumoniae blood isolates. Microbiol Res. 2005;160(2):135-40.

6. Gulmez D, Woodford N, Palepou MF, Mushtaq S, Metan G, Yakupogullari Y, et al. Carbapenem-resistant Escherichia coli and Klebsiella pneumoniae isolates from Turkey with OXA-48-like carbapenemases and outer membrane protein loss. Int JAntimicrob Agents. 2008;31(6):523-6.

7. Poirel L, Yakupogullari Y, Kizirgil A, Dogukan M, Nordmann P. VIM-5 metallo-beta-lactamase-producing Pseudomonas putida from Turkey. Int J Antimicrob Agents. 2009;33(3):287.

8. Coque TM, Baquero F, Canton R. Increasing prevalence of ESBL-producing Enterobacteriaceae in Europe. Euro Surveill. 2008;13(47):5437-53.

9. Pitout JD, Laupland KB. Extended-spectrum beta-lactamaseproducing Enterobacteriaceae: an emerging public-health concern. Lancet Infect Dis. 2008;8(3):159-66.

10. Czeizel AE, Rockenbauer M, Sorensen HT, Olsen J. Augmentin treatment during pregnancy and the prevalence of congenital abnormalities: a population-based case-control teratologic study. Eur J Obstet Gynecol Reprod Biol. 2001;97(2):188-92.

11. Weber DJ, Tolkoff-Rubin NE, Rubin RH. Amoxicillin and potassium clavulanate: an antibiotic combination. Mechanism of action, pharmacokinetics, antimicrobial spectrum, clinical efficacy and adverse effects. Pharmacotherapy. 1984;4(3):122-36. 


\section{Beytur A et al.}

12. Rodriguez-Bano J, Navarro MD, Retamar P, Picon E, Pascual A, Extended-Spectrum Beta-Lactamases-Red Espanola de Investigacion en Patologia Infecciosa/Grupo de Estudio de Infeccion Hospitalaria G. beta-Lactam/beta-lactam inhibitor combinations for the treatment of bacteremia due to extended-spec- trum beta-lactamase-producing Escherichia coli: a post hoc analysis of prospective cohorts. Clin Infect Dis. 2012;54(2):167-74.

13. Beytur A, Yakupogullari Y. A case of chronic prostatitis presenting with high Psa levels as a result of ESBL-producing E. coli. $J$ Turgut Ozal Med Cent. 2011;18:119-21. 